\title{
Article:
}

\section{Assessment in Child Protection}

Social worker's voices in England and Norway

by

Vibeke Samsonsen

PhD student

University of Stavanger

Email: vibeke.samsonsen@uis.no

Elisabeth Willumsen

Professor

University of Stavger

Email: elisabeth.willumsen@uis.no

\section{Keywords:}

Accountability, assessment model, Child Protection, England-Norway, professional judgment, social workers 


\begin{abstract}
Good quality assessment in Child Protection is crucial to ensure adequate protection and provision. This article explores social workers experiences with two different Child Protection assessment models: the "professional judgment model", exemplified by Norway, and the "structured assessment model", exemplified by England. The aim is to explore the experiences of social workers who carry out assessments in England and Norway, and compare and discuss these experiences in light of "accountability" theory.
\end{abstract}

Fourteen Child Protection social workers were interviewed about their experiences with assessment. A thematic analysis revealed three main themes that are the focus of the comparison: 1 . assessment structure, 2. professional judgment, and 3. context.

Social workers in Norway see professional judgment as a core element of Child Protection assessment processes, but would like a more structured framework to help them to make good judgments. However, they are fearful of excessive bureaucracy and a mass of paperwork, and they are anxious about having less time to support families.

While the social workers in England are proud of their triangle model of assessment and the thoroughness of their structured system, they long to be trusted more in using their professional judgment, and for more resources to be available to meet the needs of families.

These findings are discussed in light of contextual factors, and analysed in relation to the concept of accountability: How does the government in each country restrict and support social workers` professional judgment?

This study indicates the need for both an adequate structured assessment model and an emphasis on reflective processes in the use of professional judgment. The study also highlights how crucial contextual factors such as resources and public trust are in enabling good quality assessments. 


\section{Assessment in Child Protection}

\section{- social workers' experience in England and Norway}

Over the last 50 years, the focus on abuse and neglect has increased in the Western world. National Child Protection and Child Welfare are organized differently in different parts of the world because they are "social configurations rooted in specific visions for children, families, communities and societies" (Cameron \& Freymond, 2006). However, a common feature in Child Protection systems is the process of early assessment after a referral of concern is received. At its simplest, the process of assessment refers to the gathering of information to provide the basis for decision-making, planning and resource allocation (Kirton, 2009). One of the most controversial and complex areas in Child Protection is the assessment of a child and their family in terms of risk and need (Holland, 2009). Since 2000, the number of referrals to Child Protection has increased in several Western countries: Australia, Canada, the US, England and Norway (Kirton, 2009; Studsrød et al., 2012). This highlights the importance of assessing the "right" referrals, and the need for good quality assessment to reveal and prevent child abuse and neglect. Several assessment models and procedures have been developed, and most Western countries (e.g. Australia, the US) have chosen risk assessment models that can be defined as: "The systematic collection of information to determine the degree to which a child is likely to be abused or neglected in the future (English \& Pecora, 1994). Risk assessment models have been chosen on the basis of public debates, which have followed several tragic deaths of abused and neglected children in the respective countries.

The "professional judgment model" is primarily a Scandinavian social democratic model. It is characterized by few guidelines and a strong emphasis on children's and families' needs. There is little focus on the type of risk assessment evaluation that is the focus in England, which has one variation of a risk assessment model. The assessment models in the two countries differ greatly in terms of assessment procedures. The recent Munro Review in England (2011) emphasized the need to refocus on social work and professional judgment in assessments, as well as the fact that English social workers spend too much time on procedures. An equivalent report in Norway (Report of Auditor General of Norway, 2012) stated that an overemphasis on professional judgment, with too few procedures, may be a problem in Norwegian 
assessments, in part because the services offered differ greatly in their organization and content, both between social workers and between municipalities.

This article explores the experiences of social workers conducting assessments in England and Norway. These experiences are compared and discussed in light of "accountability" theory, and we aim to find out: What can we learn from these different assessment practices?

Earlier research documents the division between liberalistic "Child Protection" systems and social democratic "Child Welfare systems" in terms of "risk" and "need" (Khoo, 2004; Parton \& Skivenes, 2011). The number of Norwegian studies of assessments is limited, and the Scandinavian literature mostly focuses on the service user`s perception of assessments (Uggerhøj, 2011). Thus, in this study, we present a comparative contribution to areas that are not well documented, such as differences in governmental support for- and restrictions on professional judgment, in addition to contextual factors that affect assessment such as public debate and resourcing. This study provides opportunities to study assessment as a social work practice in two different countries, contrasting and comparing different practices.

First, we present some basic information about assessment in the two countries, and introduce the concepts of professional judgment and "accountability" in relation to assessments. After a brief presentation of our research method, we present the empirical findings in three themes:

\section{Assessment structure \\ 2. Professional judgment \\ 3. Contextual factors}

Thereafter, we discuss the findings in terms of accountability, and reflect on the level and form of governmental support provided to social workers conducting assessments. Lastly, we provide a conclusion on what we can learn from this comparative study. 


\section{Background}

\section{The English assessment framework}

As a result of serious cases of abuse and neglect, England has implemented national procedures for assessment in Child Protection. Between 1970 and 1985, 35 public inquiries were conducted in relation to cases of serious child neglect or abuse by their caregivers, in which the Child Protection system failed to reveal and prevent the mistreatment (Bochel et al., 2009). This led to extensive public debates, and social workers were criticized for not recognizing the symptoms of child abuse, and for putting too much of an emphasis on cooperating with the adults at the cost of the children. The UK Department of Health introduced the publication, "Protecting Children: A Guide for Social Workers Undertaking a Comprehensive Assessment" (Department of Health, Department for Education and Employment and Home Office, 2000), which followed the introduction of the "Children Act" of 1989. The new assessment framework was designed to "provide a systematic way of analysing, understanding and recording what is happening to children and young people within their families and the wider context in which they live" (ibid., cited from Department of Health, Department of Education and Employment and Home Office, 2000 p. 8). This is the basis for the current assessment model, "the Assessment Framework". Following the tragic death of Victoria Climbie in 2000, the public inquiry led by Lord Laming (see the Laming Report, 2003) resulted in the "Every Child Matters" policy. This rearrangement of social services was one of the biggest social political reforms in England (Simon \& Ward, 2010), with the main aim that children's care should be "everybody's business".

The Common Assessment Framework (CAF) came as a result of the "everybody's business" approach, and is designed to promote early preventative intervention that coordinates assessment across multiple childcare professionals. A simple assessment form is used, and it is possible for other professionals and agencies to identify and register whether a CAF has been completed (Holland, 2009). This is a "lighter" form of assessment than the more thorough version undertaken by the Child Protection system, and there is also a distinction between what is called the "initial" and "core" assessment within Child Protection, according to the time and depth of the assessment. Core assessment is the thorough, in-depth assessment based on information gathered through "the Assessment Triangle" (Fig. 1). The guidance for 
this model is evidence-based (Holland, 2009), and the triangle consists of three equally important elements: the child's developmental needs, the parenting capacity and family and environmental factors. As the figure shows, every side of the triangle has further specific sources of information and issues to be investigated. In addition to this triangle, national and local procedures are developed, as well as computer systems, including timescales and mandatory written reports. Hence, English Child Protection assessments constitute a structured model that emphasizes procedures and specifically designed computer systems.

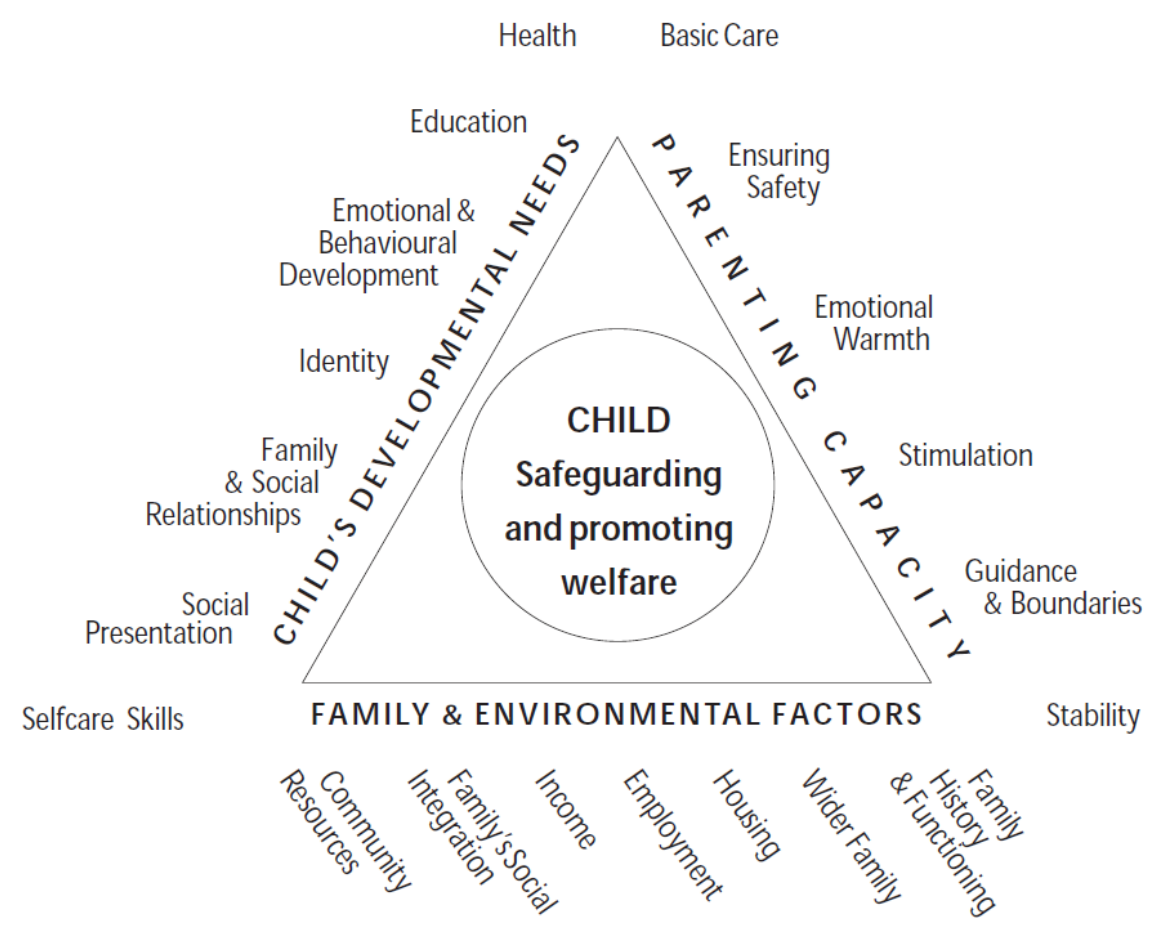

Figure 1: The Assessment Framework

Source: Department of Health (2000a: 17).

\section{The Norwegian assessment framework}

Historically, Norway was the first country in the world to establish a public Child Protection/Welfare system (Stang-Dahl, 1978). Unlike the English "risk-based" model, the Norwegian system is centred on children's and families' broad needs for services and interventions (Christiansen, 2011). In the 1980s, the Norwegian Child Protection system was criticized in the media because of children receiving insufficient help after referrals: the so called "folder children". This public debate has influenced how the system handles referrals and assessments, with timescales enacted in the Child Welfare Act of 1993 (Bunkholdt \& Sandbæk, 2008). The main assessment guidelines are the basic principles of the Child Welfare Act itself, 
combined with a deadline of three months for completion. The principles are: "in the best interest of the child", combined with the "least intrusive act" and "the biological principle". As these principles show, Norway does not have an explicit assessment model or mandatory procedure. This underpins the idea that professional judgment is the primary component when social workers assess referrals based on concern or when the child appears to have a "special need for interventions and support" (Kane, 2006). Moreover, there are no specific national guidelines or procedures for carrying out Child Protection assessments in Norway.

However, municipalities have the power to implement Child Protection assessment frameworks independently for the purpose of structuring the information gathering process on the basis of risk factors (Kvello, 2011), which has resulted in local variations throughout the country. The Child Protection office at the local level has the opportunity to consider the best way to investigate concerns, meaning that the amount of information gathered and the extent of family contact will depend on the specific situation and professional judgment of those involved. Municipalities often develop procedures in cases where there are major concerns such as child abuse, and although it is not mandatory to write a final assessment report, a report is often produced or written in the child's journal. In summary, the Norwegian assessment model is characterized by "professional judgment" because there is no common framework for structuring assessments and no standard national procedures. Although the "Children's Act" sets timescales and provides some juridical principles, assessment is still carried out on the basis of the social worker's professional judgment and according to municipal frameworks.

\section{Assessment in England and Norway - contextual factors}

In terms of preventing and detecting child abuse, the governmental response in England over the last two decades has been through risk assessments and bureaucratization (Munro, 2011). The Norwegian governmental response to criticism of its Child Protection service has been to transfer more resources into the system in the form of staff, interventions and continuing professional development for social workers already employed (NOU, 2000:12 p 111). The terms "risk", "need" and "abuse" may be regarded as socially constructed phenomena, in which the content is culturally and normatively defined (Parton et al., 1997). This implies that a country's 
social policy reflects its values, and in the case of Child Protection, these values have an impact on how social workers carry out assessments (Bochel et al., 2009). The ideal of Norwegian social democracy is based on solidarity and a high degree of government intervention, whereas the more liberal English system, is underpinned by values of personal privacy (Gilbert et al., 2011; Kirton, 2009). The Child Protection literature is commonly divided between "Child Protection" in the liberal Western countries (e.g. the US, Canada and England) and "Child Welfare" in a social democratic context (e.g. Norway and Sweden). Traditionally, "Child Protection" systems focus on risk assessment, while "Child Welfare systems" tend to have a more therapeutic orientation towards families' needs (Christiansen, 2011; Khoo, 2004).

The child population in England consists of 13,000,000 children, with approximately 50-100 of these children dying every year because of mistreatment (Kirton, 2009). Terrible tragedies such as the death of Victoria Climbie and Peter Connelly (Baby P) have been extensively debated in the English media. The government response to these debates has been to implement major reviews of services, which in turn led to social policy reforms with direct impacts on Child Protection assessments. The child population in Norway consists of approximately 1,120, 000 children (SSB, 2012), though the authors have not succeeded in identifying the estimated number of child deaths due to abuse in Norway, but it certainly occurs. One tragic event was the death of 8-year-old Kristoffer Kihle Gjerstad, who was beaten to death in 2005. His stepfather has been convicted of the killing, which led to a public debate about the responsibility of children's services. However, unlike the debate in England, this Norwegian debate did not lead to a national review of services, responsibilities and an identified need for change.

The public debates in England, especially in relation to the deaths of Victoria Climbie and Baby $\mathrm{P}$, almost took the form of a witch hunt against the social workers, doctors and managers involved. Several people were sacked or resigned from their jobs, and their names and faces were on the front pages of national newspapers and magazines, as well as on the radio and TV news. In Norway, the public debates related to the death of Kristoffer Kihle Gjerstad did not reach this personal level. No individual, other than the killer, was directly blamed except for Kristoffer`s mother, 
who was convicted of not protecting her son. Kristoffer's grandmother has led the debate, asking: "What can we learn from this? How can we prevent this from happening again?" (Gangdal, 2010). Green (2008) provides a thorough analysis of the differences in public debates between Norway and England; different political cultures and the structures that sustain them create different incentives to respond to crimes. In England, both majority parties have been impelled to respond loudly and clearly to high-profile cases. Any opportunity to exploit weaknesses in political opponents is used for one's own party gain. In contrast, Norway has a multi-party system based on consensus and compromises, and there are fewer incentives to attack political opponents. Crimes are less likely to become a means to gain political capital than in England. When it comes to the media, there is a highly competitive press market in England, with the need for catchy headlines, and less trust in expert comments on cases. This is not the case in Norway, where even the tabloid press presents a wide array of views of claim makers including experts, which has led to more balanced reporting and discussion (Green, 2008). There appear to be differences between the public debates in England and Norway, particularly with regard to cultures of blame and responsibility.

In many respects, a recent review of Child Protection in England (Munro, 2011) is very different from earlier reviews of Child Protection (Parton, 2011). It stresses the need to refocus social work and professional judgment in assessments, while also emphasizing that English social workers spend too much time on procedures (Munro, 2011). This involves moving from a system that has become over-bureaucratized, with a focus on compliance, to one that values and develops professional expertise and focuses on the safety and welfare of children and young people (Munro, 2011). Parton (2011) calls this an attempt to bring about a paradigm shift in English Child Protection. By contrast, a recent national report in Norway states that too much of an emphasis on professional judgment and too few procedures may be a problem in Norwegian assessments, partly because Child Protection services differ significantly between municipalities and between different social workers (Report of Auditor General of Norway, 2012). This report showed that a large number of shelved referrals across the country were evaluated as requiring an assessment when they were reviewed by other social workers. Thus, from the ongoing debates in England 
and Norway, we see the pendulum swinging between risk assessments/procedures and professional judgment.

\section{Theoretical approach}

\section{“Accountability" and professional judgment in Child Protection}

Professional judgment, also known as discretionary work, is a clinical consideration based on intuitive evaluations informed by knowledge and practice (Hanssen et al., 2010). The discretionary powers of welfare state professionals can be troublesome in different ways: They can threaten the predictability, legality and equality of treatment, which raises some democratic issues concerning public control (Molander et al., 2012). A metaphor for this discretionary power is "the black hole of democracy", describing the lack of public "control" over decisions based on professional judgment (Rothstein, 1998; Eriksen, 2001). The tensions of professional judgment cannot be removed, only ameliorated (Molander et al., 2012), but discretionary work can also be seen as an "opportunity" in the way it designates room for autonomy in judgments and decisions (ibid.). The delegation of professional judgment is based on trusting the willingness and ability of professionals to make good decisions (Molander, 2013). As a professional group, social workers are trained to handle general rules based on knowledge, but these general rules do not cover all the decisions related to individual needs that may be necessary for a social worker to act, e.g. "in the child's best interest". This indeterminacy creates room for normative personal evaluations and decisions (ibid.). "At the heart of the humane project of social work are a range of informal, moral rationalities concerning care, trust, kindness and respect. These rational aspects of practice create a range of practical-moral dilemmas that are difficult to systematize" (Broadhurst et al., 2010, p. 1046). At the same time, extensive research shows how heuristics leads to biases and faults in human professional judgment, as people tend to reduce complex tasks of assessing probabilities and predicting values to simpler judgmental operations based on a limited number of heuristic principles (Tversky \& Kahneman, 1974). One argument for professional judgment in social work is the need for flexibility and an adjustment to individual needs and situations. An argument against the use of professional judgment is the possibility of arbitrariness and/or poor decisions based on biases. However, eliminating professional judgment in professions such as social work and 
Child Protection is not an option because it is not possible to make rules that cover such complex situations (Molander, 2013).

The concept of "accountability" has leapt to prominence and become identified with one of the core values of democratic governance (Mulgan, 2003). Accountability in relation to professional judgments is connected to a process in which the professionals are made to be responsible for their decisions and actions, and this is seen to be a method of keeping the public informed (ibid.). A synonym could be "answerability", thus illustrating the need for public control with regard to professional judgments (Molander, 2013). There are different ways a government can make a profession accountable. One main distinction is between structural accountability, targeting the space for professional judgments; another is epistemic accountability, focusing on reasoning and reflexivity as the basis for professional judgment. The primary goal of structural accountability is to restrict the space for professional judgment, whereas the main objective of epistemic accountability is to improve the conditions for professional judgment (ibid.). In Child Protection, examples of mechanisms for structural accountability are laws, regulatory agencies and the fragmentation of tasks within the organization. The mechanisms for epistemic accountability in Child Protection are the formal education of social workers and more support systems, such as supervision.

We can discuss the debates in Child Protection in Norway and England in light of accountability. For example, when criticizing the over-bureaucratization of the English assessment model, the Munro review emphasizes the need to reduce the mechanisms of structural accountability in order to enhance epistemic accountability, thereby underpinning the need for more reasoning and reflectivity in Child Protection (Munro, 2011). Broadhurst et al. (2010) argue that English Child Protection practice is at a crossroads, and that the government is willing to acknowledge that improvements cannot simply be made at the level of organizational structures, but that there is a need for a re-professionalization of social work. The Norwegian Riksrevisjon`s Report (2012) cites the problem of extensive variation in the level of services across Norway, which may substantiate the need for a more structural accountability in Child Protection so that the present epistemic accountability can be more efficient. 


\section{Methods}

A qualitative research design was chosen to explore social workers' experiences of assessment. Qualitative methods are usually perceived as helpful for collecting material for in-depth analysis, as we have done in this study (Patton, 2002).

\section{Sample and analysis}

The main data source for our analysis was a series of interviews with social workers who were conducting assessments in Child Protection in Norway and England. Fourteen social workers in total (Norway=8, England=6) were interviewed for approximately one hour each, using a semi-structured interview guide. The sample was recruited through the city councils/municipalities in Bergen, Norway $(258,000$ inhabitants) and Bristol, England (433,000 inhabitants). Both cities have wellestablished local authorities, as well as the social workers recruited work in the Child Protection services in the two municipalities that constitute the selection criterion. The social workers interviewed were qualified social workers with at least three years of work experience.

The questions concerned the social workers`experiences with the assessment work and their points of view regarding assessments, e.g. what promotes and what inhibits good quality assessments. A pilot study was conducted before the data collection started, in which two social workers, one from Norway and one from England, were interviewed. The pilot study helped us formulate the interview guide and shape the research focus in data collection. All the interviews were transcribed analysed using a stepwise structured thematic analysis as described by Braun and Clarke (2006). The findings were presented and discussed with a range of research fellows on several occasions. Thematic analysis is a way of analysing data to identify and report patterns and themes (Braun \& Clarke, 2006), and we familiarized ourselves with the data in different ways: By reading and re-reading, by comparing within countries and between countries and by searching for themes within the material, coding these themes and naming them. We then critically reviewed the themes emerging from the material by searching for statements from the material that would support and illustrate the themes. Throughout the process, it has been very important for us as researchers to be "faithful" to the aims of the study: to explore social workers ' own experiences, perspectives and views regarding assessment in Child Protection. 
During this process, we have also discovered new and unexpected themes in the material, such as the presence of emotions in the English dataset. Overall, three main themes were identified and chosen for analysis according to our research aim:

\section{Assessment structure; \\ 2. Professional judgment; \\ 3. Contextual factors.}

During the analysis, further categories were identified under each of the three main themes: "assessment structure" includes an "assessment framework/model" and "procedures", "professional judgment" includes "discretion" and "reflectivity", while "contextual factors" includes "public debates" and "resources". The final analysis involved organizing a "thematic map", with connections between themes and categories illustrated with arrows and text. The findings presented in this article are based on this thematic map, and the quotes presented are marked according to the social worker being interviewed (e.g. E1=English participant 1, N1=Norwegian participant 1).

\section{Methodological reflections}

Cross-national research offers opportunities in patterns of differences and similarities between countries and, together with contextual factors, this gives us new perspectives and contrast in our search for knowledge (Ragin, 1994). In our study of assessment as a social work practice across two countries, the two different ways of practicing offer opportunities to reflect on differences and similarities (Baistow, 2000). However, is the phenomenon comparable in the two countries? Our sample is recruited from one city in each country: Bristol in England and Bergen in Norway. Then to what extent can these two cities represent national ${ }^{1}$ contexts for comparison? Although there is municipal freedom concerning implementing assessment tools in both Norway and England, we consider that in this study they represent the overarching assessment models labelled "the structured model" (England) and "the professional judgment model" (Norway). We identify differences between assessment tools within each of the two countries, but we also find data

\footnotetext{
${ }^{1}$ In a strict sense, because it is part of the UK England is not a nation/country. However, within the UK there are different models concerning the Child Protection assessment. In this article, we choose to focus on the model in England.
} 
material from Bristol and Bergen to help illustrate differences in contextual factors between the countries that may explain such differences. This is not an attempt to provide a causal-effect analysis of assessments. Instead, our concern is how to generalize our finding in relation to our research question based on a relatively small sample in this study. Nevertheless, we assume the results may provide valuable knowledge in contributing towards improving child protection services across countries, which was the overall purpose of the study.

Ethical approval was obtained from the Data Inspectorate of Norway (ref. no.29993) and the Board of Ethics at the University of Bristol, and managers in both city councils/municipalities have approved the study. Written consent was provided by the participants, who were assured that all information would be treated confidentially, and that they were free to withdraw from the project at any time and have their statements deleted. All data were rendered anonymous, and will be deleted at the end of the project.

\section{Findings}

\section{Introduction}

We have divided the description of the empirical findings into three sections, representing the three main themes in the analysis. We begin each section by presenting the English findings, and then follow with the Norwegian findings.

Theme 1: Findings regarding assessment structure

Assessment as a structure was divided into two main categories in the analysis:

\section{Assessment structure as a specific framework/model;}

Assessment structure as procedures.

\section{England}

"Thorough bureaucracy" constitutes a core description of how the English social workers viewed their existing assessment framework. They described the assessment model as consisting of the Assessment Framework (triangle) and the procedures to be followed. 


\section{1: Assessment framework}

The assessment triangle is unanimously referred to in a positive manner:

E3: "For me, the triangle is a point of reference to make sure everything is covered. Some form of memory aid almost, so I will go out with that in mind that I need to cover all these issues."

E1: "The assessment triangle reflects what lies at the heart of our assessment process. We will never lose that because I think it is extremely helpful and useful."

Despite the positive attitudes toward the triangle in terms of its usefulness for gathering information and covering all the issues, it does not provide "answers" with regard to difficult decisions that have to be made:

E3:"I don't think it helps me necessarily to come to a decision. It helps me gather information, but it does not help me analyse it."

\section{2: Procedures to be followed in assessment}

The English social workers interviewed talked about the number of procedures that had to be followed when making assessments, both national- and local procedures. When it comes to these procedures following the assessment triangle, the comments are more negative than those about the triangle as an assessment framework. Typical verbs used were "over-complex" and "box-ticking".

E1: "The reality is you sit there with this enormous thick thing of guidelines. It can completely freeze your mind. There is an awful lot of information to read about how to carry out an assessment, guidelines, etc. It is complete overkill, but it is a response over things that happened in the past and the need to make sure every last corner is covered."

Some of the social workers have different assessment experiences from other districts, both better and worse, and they would say that this is partially due to variations in local procedures. The computer system seems to be especially important with regard to how they view local procedures for assessment. Bristol City 
Council recently changed their computer system, and this was a theme across the interviews. The social workers stressed the importance of a functional and helpful computer system in assessment: To register information, to write reports, and most important from their point of view, to support reflective thinking and decision-making, and not restrict them to "boxes", i.e. where computer systems fragment the information into time-consuming boxes to be ticked, hence making it difficult to keep the holistic view of the family.

E2: "I cannot get the flow, and I cannot get my ideas down, because I am too busy thinking about the boxes. What is the parent capacity and so on. They are all interwoven in some way, so how do you separate them? It is fragmenting."

An ongoing theme among the English social workers is the wish to be able to write in a more flexible document, without having to fit in with the boxes that have to be ticked.

E5: "So, if I was in charge I would literally just have the headings, and you got to be creative in how you use it. It feels like it is ticking boxes rather than... It takes too long. You have got the information, and you just want to write it down so it makes sense, and have an analysis in pulling everything together."

In sum, the English social workers interviewed found the Assessment Framework (triangle) to be helpful; however, they felt that the guidance following assessment to be overwhelming, and the box ticking in the computer system to be fragmenting.

\section{Norway}

"It depends" constitutes a core description of how the Norwegian social workers approach assessment. They present their existing model as a (national) lack of a specific framework (although recent locally implemented framework) and office "procedures".

\section{1: The assessment framework}

N1:"The assessment process will depend on the referral. Is it sexual abuse or violence? How are we going to approach it? Who is it natural to invite to the first 
family meeting? It depends on the age of the child, whether the parents are living together..."

N7: "We start with a meeting with the parent. It all depends on the referral."

As previously described, Norwegian social workers conducting assessments do not have an explicit assessment model or many procedures in relation to assessment. The municipality of Bergen has recently implemented an assessment model called "Kvello". The social workers interviewed were all part of this implementation, but were "free" to choose whether they want to use this model from case to case. However, they do not distinguish between the use of "Kvello" and not using this framework when describing the "it depends" category. The main difference is the information gathered. The "Kvello" model functions in a similar way to the English triangle in prompting the social worker about the areas of information to gather in assessments. The Norwegian social workers interviewed welcomed this structure, and overall they appreciated the ability of the model to systematize information. This "it depends" way of assessment was described in terms of the possibilities for creativity and the lack of standardization as in set standards and processes. The Norwegian social workers talked about "travelling ideas": Ways of approaching or structuring assessment that occurred to them as a result of a good idea passed on by a colleague from another office, or from experience in another district office using different procedures. Two "travelling ideas" mentioned were: the possibility of having a meeting when gathering information instead of writing letters to ask for information as they usually do, and the possibility of using written assessment plans to inform/plan together with the family in the assessment process.

\section{2: Procedures to be followed in assessments}

When it comes to assessment, there does not seem to be many specific procedures to be followed. The social workers refer more to juridical principles such as the duty of confidentiality when talking about procedures in assessment. A common feature in the Norwegian interview material was talk about "office procedures". These are procedures that apply at a level below the local level. Bergen would be a local level, but the procedures developed are office-based (Bergen has eight Child Protection offices). 
N4: "We have something we do regularly. I have never gotten a set formula, but the way we do it in our office is to have a first meeting with the parents presenting the referral. Then gather information, do home visits, observe. We agree to always meet and talk with the child."

Even though the social workers refer to these procedures as office procedures, one can see that the procedures are quite similar between different offices. Concerns regarding sexual abuse and violence seem to have more explicit procedures at the office level. Additionally, the Norwegian social workers stressed how computer systems influence their ability to perform good quality assessments, and how the computer system can hinder them and be time consuming. The new "Kvello" framework was mostly commented on in relation to the computer system accompanying the implementation of the model. At best, the new computer system was viewed positively in terms of its systematization of information, but it was also viewed negatively in terms of being too time consuming because it lacks the ability to duplicate information in different documents.

So in summary, the Norwegian social workers viewed their assessment framework as offering possibilities for choosing different approaches and methods depending on the features of the case. But on the basis of statements about the new "Kvello" model that had been implemented locally, it seems as if the social workers are welcoming more structure in their assessment, particularly in relation to information gathering. They currently refer to few procedures when they carry out assessments, and barely know where to find written procedures.

Theme 2: Findings regarding professional judgment in assessment

The categories of discretion and reflectivity in assessment are part of one theme because both aspects relate to clinical considerations. Professional judgment was previously described as clinical considerations based on intuitive evaluations informed by knowledge and practice. In this context, reflectivity refers to the analysis and critical thinking that takes place before deciding the direction of a case. It has been difficult to separate the terms of discretion and professional judgment, although professional judgment in this study is a more overarching concept that also includes 
reflectivity processes. When analysing the empirical material, we divided this theme into two categories:

\section{Discretion in assessments; \\ Reflectivity in assessments.}

\section{England}

\section{1: Discretion in assessment}

In England, the terms professional judgment and discretion are not frequently used when talking about Child Protection assessments. The English social workers varied in their perception and attitudes toward professional judgment and discretion, and viewed the concepts as complex.

E6: "I think professional judgment is controversial. I think it is really important, and I think it is important to value experience. It is controversial when you have people who think their professional judgment overrides everything. It is, and has been, undervalued, but also our society is terrified of litigation. So everything is about let's cover our backs, and I think that is why we are so bureaucratic. People can get sued or... Professional judgment is going to be a big cultural shift for people."

E1: "Regardless of the complexity of the form we have to complete, in the end we have to produce a summary and an analysis, so at the end of the day, on the basis of all the information you have pulled together, you have to form some sort of professional judgment about it".

Professional judgment is explicitly separated from the process of personal judgments by most of the English respondents, e.g.:

E5: "A professional judgment is very different from a personal judgment. That is because you are going on the guidelines, the law, your experience, what you know about risk and evidence, research, things like that." 


\section{2: Reflectivity in assessment}

Although every English social worker interviewed regarded reflectivity in assessment as crucial for good quality decisions, they differed in how they presented and viewed the level of reflectivity in their workplace when conducting assessments. Two of the six social workers were very pleased with the level of reflective support and discussion, describing this as a reflective office culture with open doors. The other four English social workers complained that there was not any room for reflectivity in assessment, and only in case management. The following two quotations illustrate both experiences regarding the level of reflectivity:

E4: "I think we are lucky with the management which we have. Her door is always open, and you can change your mind. We are very much allowed to talk about every bit of that, so it is a sort of thought process. So I think having that opportunity to talk through decisions... I am lucky, but I know that not everybody is."

E2: "There is not a culture of reflection in our team, there is not much space. We are trying to improve it and to take ownership, because we need to be reflecting on the work, but it is much about case management and case directions. What would be really useful to have is the good quality reflections in supervision, but there is not the space or capacity in my team, and my view is that it is not good enough actually."

The social workers who were not pleased with the level of reflectivity in assessment referred to this as a non-reflective office culture, and related this to high workloads and pressure. One of the social workers stated that she totally understands why reflective supervision is not possible in her system, with her manager being responsible for 350 children.

In summary, the English social workers showed ambiguities regarding professional judgments, in particular discretion in assessments. They acknowledged professional judgments to be a central part of assessments and, at the same time, they considered the term to be controversial. Reflective thinking in assessment was viewed as very important in decision-making. However, the social workers' experiences regarding reflectivity differed between offices. 


\section{Norway}

\section{1: Discretion in assessment}

The Norwegian social workers commented on discretion and professional judgment in their assessment: "That's what we do when assessing." They stressed how professional judgment is required and supported in the Child Protection system, and how this judgment is a "red line" throughout the assessment from receiving the referral to concluding the assessment.

Words they use to describe what professional judgment consists of are "gut feeling", "personality" and "subjectivity", all of which are informed by their knowledge and previous experiences.

N3:"Professional judgment is about our pre-understandings regarding the specific issues in the case, and how we use our knowledge. It is often subjective."

N2: "It is always complicated and you never get a set formula. You have to start with what the parents are expressing, gather information, see what the children express, and then it depends on yourself actually, how you are feeling, thinking... A bit of professional judgment and a bit of gut feeling."

But some of the social workers raised objections to the high level of professional judgment involved in making the assessments.

N6: "Professional judgment in making assessments for me is the fact that every social worker has to make some choices about how to conduct an assessment. Even though every assessment is about assessing, I think there are huge differences between assessments. Some assessments being too thorough, others too superficial, not revealing the problems and issues".

Almost every Norwegian social worker interviewed linked professional judgment and reflectivity, considering that good quality judgments are based on reflecting on the case together with other professionals. 
N1: "You have to evaluate every single case on the basis of the information you have got, and fortunately most often there are two social workers assessing together, so we can discuss the case. And the other social worker might see something different."

In summary, the Norwegian social workers also show ambiguities about the use of discretion in assessment, but in a different way from the English social workers. In Norway, discretion seems not to be controversial in assessment, but some of the social workers problematize the amount of professional judgments in assessments, especially the huge variation in practicing assessment and the lack of universal standards leading to quality variations.

\section{2: Reflectivity in assessment}

In the Norwegian interviews, the social workers did not explicitly separate the concepts of professional judgment and reflectivity. The two concepts seem to be viewed as part of the same "tailoring"/individual approach to assessment. This quotation illustrates the perceived connection between reflectivity and professional judgment:

N3:"There are several fora to discuss the decisions: Supervision once a week, discussions with your team, other colleagues and the head manager. So, we always have other people to talk to, and we don't have to make difficult decisions alone, and in my opinion that is very important and is related to professional judgment, how we view things differently."

In the Norwegian interviews, all eight social workers described reflectivity through various discussions in several fora as a matter of course in assessment to "quality check" professional judgment.

In summary, both professional judgment and reflectivity seem to play a key role for social workers conducting assessments in Child Protection in Norway.

Theme 3: Contextual factors influencing assessment

The third main theme, "contextual factors influencing assessment", is coded in two categories: 


\section{Public debates related to the role of social workers;} Available resources.

Our findings support the traditional division of "Child Protection" and "Child Welfare". All the social workers interviewed in England used the term "risk" when describing the assessment process. Only one out of eight Norwegian social workers used the term risk, while the other seven talked about fulfilling the child`s and the family`s "needs". The English social workers talked about uncovering abuse, while the Norwegian social workers referred more to therapeutic intervention to prevent future damage, and help fulfill the psychological needs of the child. However, in our analysis, we choose not to focus on this aspect, because this has been well documented in previous research (Khoo \& Nygren, 2002; Parton \& Skivenes, 2011). Our interview material has additional richness with regard to social workers` description of other contextual factors influencing their assessments, such as public opinion and the available resources.

\section{England}

\section{1: Public debate related to the role of social worker}

As described above, the debates about Child Protection in England have been very influential in creating today's system. The children who have died while under Child Protection and the media debate that followed have had a major influence on the assessment process:

E2: "There is a real culture of people hating social workers in the UK. We are the enemy, and that's the way it is seen, and that's the way the British media portrays social workers, and the fact that whenever there is a child death the social workers will be more blamed than the police."

E6: "There is not a day goes by that you don't hear the phrase, "you have to make sure you have covered that, just in case this happens or somebody accuses you of that", and it can really inhibit good practice. I think the media has a lot to do with that, it is very media driven. It is a witch hunt culture, which is horrible. People want someone to resign whenever there is a crisis or an accident. I go into work every single week and think that could be me all over the newspaper." 
All the English social workers referred to the way public opinion towards social workers influenced their professional and personal life. They experienced fear and anxiety about the possibility of failing to recognized risky situations professionally, and personally, for instance, they tended not to present themselves as social workers at parties to avoid the personal stigma connected to the role.

\section{2: Recourses available:}

Traditionally there is a difference in public resources between the social democratic policy systems and more liberal policy systems (Kirton, 2009). The Scandinavian "Child Welfare" system, of which Norway is a part, is based more on the redistribution of resources through higher taxes than the more liberal English "Child Protection" system. The findings seem to reflect this. In our material, social workers in England were generally frustrated that when service users' needs were revealed through assessment, they were unable to implement the necessary interventions to meet these needs. They referred to the more family-based interventions as a "resource battle" with their managers, and referred to long waiting times before the interventions took place. Some of the English social workers also described the current financial crisis in Europe as leading to visible cutbacks in family support and interventions, and they were faced with a compassion dilemma between the families they worked with and the national economy. They believed in the need for cutbacks, as stated by the government, but saw how children and families directly experienced disadvantages from these cutbacks.

E5: "For the moment, resources are difficult because we are living in this austere time, and for the next five or so years. So I understand there needs to be more cutbacks, but unfortunately that does not always meet the needs of children. There are services, but they are for people in extreme..., the thresholds are so high."

Other resource issues highlighted by social workers as influencing assessments were in relation to workload and supervision. Several of the English social workers related the lack of reflectivity in supervision to high workloads and pressured managers, illustrated by one manager being responsible for 350 children. Expressions like "being stretched" were used, and social workers talked about working until 10 o'clock in the evenings, as well as weekends. On their "wish list", the 
English social workers put more time to spend with each family in order to allow better assessments, but they did not suggest smaller workloads as a solution to help achieve this. When they were directly questioned on this issue, some of the social workers laughed, and this quote illustrates their perspective:

E4: "It would be important for caseloads to be much lower, and threshold needs to be lower as well. But we have given up..."

In summary, assessments in England are undertaken in a culture of scepticism towards social workers, within a public context of blaming individuals. In addition, limited resources and cutbacks undermine the quality of assessments.

\section{Norway}

\section{1: Public debate related to the role of social workers}

The Norwegian social workers did not refer to public debate or public opinions about social workers in Child Protection. This makes sense in a national context, where there are few debates about child deaths and a more "learning approach" towards this issue. But in our interviews they referred to perceptions of the power social workers have as an issue, in the sense that people in general are afraid of the Child Protection system and its power to remove children from their homes. The Norwegian social workers interviewed did not express any feelings of anxiety or fear about the difficult process of uncovering abuse. When it comes to responsibility or who to blame if something goes wrong, the Norwegian social workers refer to the system as opposed to individual blame.

\section{2: Resources available}

Every Norwegian social worker wanted more resources available in relation to assessments, suggesting smaller workloads and more time to spend with each family. They did not experience a national financial crisis influencing Child Protection, and were far more "demanding" in terms of wanting more resources to be made available for doing good quality assessments. They showed no concern for saving the government money, only a wish for interventions to meet the child`s and the family`s needs. 
In summary, the Norwegian assessments are barely debated at all in the media. Nonetheless, there are discussions about power and the overall system. The Norwegian Child Protection system seemed to provide interventions to meet the needs revealed in assessment. Although the Norwegian social workers would like a higher degree of individual "tailoring" of interventions, and talked about interventions as an obvious outcome of assessment in a different way from the English social workers, the feeling of being "over-stretched" because of the high workload was not expressed as desperately as in the English material.

\section{Discussion and implications of findings}

In this part, we discuss the findings presented above in light of "accountability" understood as governmental trust in the professional judgment of social workers. Structural accountability targets the space for professional judgments with a primary goal of restricting it, whereas epistemic accountability aims to improve the conditions for professional judgment (Molander, 2013). Depending on the purpose of the procedure, procedures in Child Protection can enhance either structural or epistemic accountability. The effect of mechanisms for structural accountability can have epistemic consequences, and mechanisms for epistemic accountability can depend on structures to be effective (ibid.).

The Munro Review emphasizes the need for reducing the mechanisms of structural accountability to enhance reasoning and reflectivity (Munro, 2011). Such a change may be regarded as a paradigm shift in England, and it might be too easy to target these rooted mechanisms of structural accountability by simply stating the fact that the government "allows" and wants professional judgment; thus, other contextual factors will be highly influential. Both negative public perception of social workers and their ability to make good professional judgments, and the "blame culture" on a personal level, seem to hinder professional judgment. Social workers may need the confidence created by structured accountability mechanisms because the possibility of making the "wrong" professional judgment seems terrifying. When it comes to epistemic accountability, such as governmental support to improve judgments, reflectivity is highlighted by both Munro and our informants as highly important, although the effect of reflective supervision can be questioned (Carpenter, 2013). For English assessments to be based more on professional judgments, the level of 
structural accountability may have to be reduced and replaced by more epistemic accountability, while recognizing that Child Protection is a complex field. It is almost impossible to "cover our backs" when dealing with risk in such a field. It may also be problematic to remove some aspects of the structure, such as boxes to be ticked or procedures to be followed, without replacing them with other support mechanisms, e.g. increasing the systems that support reflectivity.

In Norway, assessments in child protection are rarely debated. The Norwegian governmental accountability approach is that an epistemic accountability is superior to a structural accountability. This approach is supported by the social democratic resources available for staffing and interventions. In Norway, there appears to be a more constructive public opinion towards social workers in Child Protection, with reference to Green`s (2008) analysis of the differences in public debates. Even so, it is time to ask whether this trust in professional judgment in Norway is a bit naïve and oversimplified for such complex tasks as carrying out Child Protection assessments. This question can be raised based on the fact that clinical judgments are shown to be full of biases (Stewart \& Thompson, 2004), and there needs to be a debate about the faith in professional judgments as the "gold" standard for good quality decisionmaking.

Another issue that needs to be raised is the major differences between municipalities in Norway. In our study, the municipality of Bergen had just implemented an assessment model to improve the structure in decision-making. It seemed that the local government acknowledged social workers` need for structural accountability support to increase the quality of professional judgments, or to restrict the room for making "bad" professional judgments. However, the fact that an individual person (Kvello) has developed and initiated the assessment model implemented in several municipalities, without any national authority involved, can be questioned. This study may imply the need for a national debate and governmental decisions in Norway about a more general assessment approach. As the implementing process of the Kvello model illustrates, the lack of national debates and policy decisions about assessment models and procedures pushes this debate and decision to the local level. This demonstrates the need for more structured assessments in municipalities, 
which in turn increases casual implementation and differences between municipalities that was not the intention of the Riksrevisjon`s Report.

The debate in both countries can be enriched by comparing the two assessment models, given that the Munro Review implies the need for changes in the UK toward Norwegian standards, whereas the Riksrevisjonen's Report implies the need in Norway for more structure to support equality in services. Our study show the importance of both structural accountability mechanisms such as good assessment models and epistemic accountability with room for- and support of structures for professional judgment in assessment. Also, the assessment model has to be accompanied with recourses according to staff and interventions to be perceived as helpful from the social workers' point of view. We find the responses of different governments toward supporting social workers making Child Protection assessments to be of major significance.

\section{Conclusion - what we can learn from the comparison}

For assessment structure, the English social workers interviewed found structural support in their current Assessment Framework (the triangle). Even so, the accompanying procedures were regarded as overwhelming. The computer system led to the fragmentation of information into small boxes to be ticked, which was not seen as helpful, but rather as an obstacle to high quality assessment. The Norwegian interviews showed how assessment in Norway was built on professional judgment, with few guidelines and procedures. There seemed to be no standard process or structure for performing assessments, but approaches varied between cases and social workers. The Norwegian government has given the municipalities the freedom to determine the structure of Child Protection assessments, although direction is given on timescales and juridical principles. The Norwegian social workers mirrored this "freedom" by taking individual approaches, which was reflected in huge differences in office cultures. At best, these individual approaches contribute to "tailor-made" assessments, but overall the social workers interviewed would welcome more structure in assessment processes. They would appreciate a standard framework when gathering information. They also spoke of the benefits of planning assessments and providing a copy to the family, as well as having a final written assessment report that is currently not mandatory. 
Professional judgment in assessment seems to differ between England and Norway. The English social workers presented the concept of discretion as controversial and a culture shift for Child Protection. At the same time, they expressed the view that professional judgment is an important component of decision-making. Nevertheless, they did not want professional judgments linked with subjectivity and feelings, but to be based on knowledge and experience. The Norwegian social workers spoke of discretion and professional judgment as the main components in assessment, and expressed the concept in terms of subjectivity and gut feeling. However, social workers from both countries underscored that regardless of the model used, a decision about the direction of the case and the interventions have to be made by social workers, with professional judgment forming a large component of that decision. Across the two countries, reflectivity throughout the entire assessment process, especially in relation to decision-making, was emphasized as being crucial for good quality assessments. Our findings point to the different level of reflectivity between- and within the countries. Part of the English material suggests that reflectivity in assessments is not a central part of the process; at least it does not seem to be a standard procedure to include reflectivity in decision-making. It appears that the emphasis on professional judgment in Norway is accompanied by a culture of reflectivity, and the two concepts are interwoven.

Contextual factors influencing assessment differed greatly between the two countries. In England, a national culture of individual responsibility and blame toward social workers seemed to have a major influence on Child Protection. The social workers expressed feelings of anxiety because of the high levels of personal responsibility they would feel if they failed to prevent or uncover risk situations, particularly given how the media has handled previous cases of child deaths, identifying them as misconduct on the part of the social workers involved. This culture of blame, combined with high workloads and structural demands in the system, seemed to constitute a "squeeze" and an intolerable pressure over time. In addition, the social workers we interviewed were feeling pressured by the economic crisis in the country, thereby leading to cutbacks in already hard-pressed services for children and families. In Norway, the contextual factors influencing assessment seemed to differ a lot from the English factors. There has not been the same amount of debates in the Norwegian media, and the component of personal blame is almost 
absent in the public debates. Additionally, Norway is not experiencing an economic crisis at the moment, and the broad and well-developed services for children and families are not being cut. The workloads and demands also seem to differ, with higher workloads and longer working hours apparent in the English interviews. There are stark differences between these two systems of Child Protection shown by the themes investigated in this study, and each system has its strengths and weaknesses.

In terms of what we can learn from the study of these two different practices, we would like to highlight the importance of focusing on both the model/framework and the professional judgment component of assessments in child protection. Regarding the framework, the English over-bureaucratized child protection system might be a warning when discussing the Riksrevisjon's Report regarding the inequalities of services in Norway. Interestingly, at the moment, we register a casual implementation of a structured assessment framework (Kvello model) in many municipalities, although this is based on an individual commercialized initiative. This might be a value shift in Norwegian assessments towards a risk evaluation that focuses on structural accountability, away from the current national epistemic accountability approach, and without a national involvement to ensure the quality. However, the thoroughness and helpfulness in the English assessment triangle may serve as an inspiration for the Norwegian government when implementing a possible national framework for assessment, with the aim of achieving structural support. In England, Munro is highlighting the need for a paradigm shift in child protection. Still, this change may imply an over-simplifying in the focus on professional judgment, unless a serious discussion of epistemic contextual factors such as the "blame culture" and limited resources regarding staff and interventions are taken into consideration. This study indicates the need for supportive structures, as well as room for making professional judgments in assessments. There appear to be pitfalls on both ends of the pendulum, and the most constructive discussions on structure and professional judgment in assessment seem to be on getting the proper balance. 


\section{References}

Baistow, K. (2000). Cross-national research: What can we learn from Inter-country Comparisons? Social Work in Europe 7(3), 8-12.

Bochel, H., Bochel, C, Page, R., \& Sykes, R. (2009). Social Policy. Themes, Issues and Debates. Pearson.

Broadhurst, K., Hall, C., Wastell, D., White, S., \& Pithouse, A. (2010). "Risk, Instrumentalism and the humane project in Social Work: Identifying the informal logics of risk management in children's statutory services. British Journal of Social Work, Vol. 40, 1046-1064.

Braun, V., \& Clarke, V. (2006). Using thematic analysis in psychology. Quantitative Research in Psychology, 3 (2). pp. 77-101.

Bunkholdt, V., \& Sandbæk, M. (2008). Practical Child Protection Work. Gyldendal Akademiske.

Cameron, G., \& Freymond, N. (2006). Understanding international comparisons of child protection, family services and community caring systems of child and family welfare: Towards Positive Systems of Child and Family Welfare. pp. 327. University of Toronto Press.

Child Welfare Act (1993).

Christiansen, $\varnothing$. (2011). Children in out of home placements. PhD University of Bergen.

Carpenter, J., Webb, C. M, \& Bostock, L. (2013). The surprisingly weak evidence for supervision: Findings from a systematic review of research in child welfare practice (2000-2012). Children and Youth Services Review 35. pp 1843-1853.

Department of Health (2000a). Framework for the Assessment of Children in Need and Their Families. London: Department of Health. 
English, D .J., \& Pecora, P. J. (1994). Risk assessment as a practice method in child protective services. Child Welfare 73(5), 451-473.

Gangdal, J. (2010). Jeg tenker nok du skjønner det sjøl. Historien om Kristoffer. Kagge publishing.

Gilbert, N., Parton, N., \& Skivenes, M. (2011). Child protection systems: International trends and emerging orientations. New York: Oxford Press.

Green, D. A. (2008). When Children Kill Children. Oxford University Press.

Hanssen, H., Humerfeldt, K., Kjellevold, A., Norheim, A., \& Sommerseth, R. (2010). Professional judgment and service user participation. Norway: Fagbokforlaget.

Holland, S. (2010). Child and Family Assessment in Social Work Practice. Sage Publications.

Kane, A. (2006). Child Protection in Norway and England. Report 2006/3, Høgskolen in Harstad.

Khoo, E. G. (2004). Protecting our children: A comparative study of the dynamics in structure, intervention and their interplay in Swedish child welfare and Canadian child protection. Umeå university, Umeå.

Kirton, D. (2009). Child Social Work Policy and Practice. Sage Publications.

Kvello, Ø. (2010). Children at risk. Norway: Gyldendal.

Molander, A., Grimen, H., \& Eriksen, E. O. (2012). Professional Discretion and Accountability in the Welfare State. Journal of Applied Philosophy, Vol. 29, No. 3. 
Molander, A., \& Smeby, J-C. (Eds.) (2013). Professional studies 2. Norway: Universitetsforlaget.

Mulgan, R. (2003). Holding power to account. Accountability in modern democracies. Houndmills: Palgrave.

Munro, E. (2011). The Munro Review of Child Protection: Final Report. A ChildCentred system. Department of Education, London.

NOU 2000:12: Child Protection in Norway.

Osborne, S. P. (2002). Public management. Critical perspectives. Routledge.

Parton, N. (2012). The Munro Review of Child Protection: An Appraisal. Children and Society Vol. 26. Pp. 150-162.

Parton, N., Thorpe, D., \& Wattam, C. (1997). Child Protection: Risk and the Moral Order. Maximillian Publishers.

Patton, M. Q. (2002). Qualitative Research \& Evaluation Methods. Sage Publications.

Ragin, C. (1994). Constructing social research. Pine Forge Press.

Report of Auditor General of Norway: Document 3:15 (2011-2012). Norway: Fagbokforlaget.

Rothstein, B. (1998). Just Institutions Matter: The Moral and Political Logic of the Universal Welfare State. Cambridge University Press.

Simon, C. A., \& Ward, S. (2010). Does Every Child Matter? Routledge.

SSB: Statistics Norway, ssb.no. 
Stang Dahl, T. (1978). Child Protection and Protecting Society. PhD,University of Oslo.

Stewart, A., \& Thompson, C. (2004). Comparative Evaluation and Child Protection Assessment Tools. Queensland: Griffith University.

Studsrød, I., Willumsen E., \& Ellingsen, I. (2012). Parets` perception of contact with the Norwegian Child Welfare Services. Child \& Family Social Work.

Sæbønes, A.-M. (2006). Helsen til barnevernsbarn krever samarbeid (The health of child welfare children require collaboration). Tidskrift for den Norske Lægeforening, 126-129.

The Laming Report (2003). Official Document, government UK.

Turney, D., Platt, D., Selwyn, J., \& Farmer, E. (2012). Improving Child and Family Assessments. Turning Research into Practice. Jessica Kingsley Publishers.

Tversky, A., \& Kahneman, D. (1974). Judgment under uncertainty: Heuristics and biases.

Science, New Series, Vol. 185, No. 4157 pp. 1124-1131.

Uggerhøj, L. (2011). To be assessed. Norway: Universitetsforlaget. 